Article

\title{
Mechanism of Proton Pumping in Complex I of the Mitochondrial Respiratory Chain
}

\author{
Jonathan Friedman ${ }^{1}$, Lev Mourokh ${ }^{1,2, *}$ and Michele Vittadello ${ }^{3,4}$ \\ 1 Department of Physics, Queens College of the City University of New York, Flushing, NY 11367, USA; \\ jonathna.friedman80@qmail.cuny.edu \\ 2 Ph.D. Program in Physics, The Graduate Center of CUNY, New York, NY 10016, USA \\ 3 Department of Chemistry and Environmental Science, Medgar Evers College of the City University of New \\ York, Brooklyn, NY 11225, USA; mvittadello@mec.cuny.edu \\ 4 Ph.D. Program in Chemistry, The Graduate Center of CUNY, New York, NY 10016, USA \\ * Correspondence: lev.murokh@qc.cuny.edu
}

Citation: Friedman, J.; Mourokh, L.; Vittadello, M. Mechanism of Proton Pumping in Complex I of the Mitochondrial Respiratory Chain. Quantum Rep. 2021, 3, 425-434. https://doi.org/10.3390/ quantum3030027

Academic Editor: Carlos F. Martino

Received: 13 May 2021

Accepted: 3 August 2021

Published: 9 August 2021

Publisher's Note: MDPI stays neutral with regard to jurisdictional claims in published maps and institutional affiliations.

Copyright: (c) 2021 by the authors. Licensee MDPI, Basel, Switzerland. This article is an open access article distributed under the terms and conditions of the Creative Commons Attribution (CC BY) license (https:// creativecommons.org/licenses/by/ $4.0 /)$.

\begin{abstract}
We propose a physical mechanism of conformation-induced proton pumping in mitochondrial Complex I. The structural conformations of this protein are modeled as the motion of a piston having positive charges on both sides. A negatively charged electron attracts the piston, moving the other end away from the proton site, thereby reducing its energy and allowing a proton to populate the site. When the electron escapes, elastic forces assist the return of the piston, increasing proton site energy and facilitating proton transfer. We derive the Heisenberg equations of motion for electron and proton operators and rewrite them in the form of rate equations coupled to the phenomenological Langevin equation describing piston dynamics. This set of coupled equations is solved numerically. We show that proton pumping can be achieved within this model for a reasonable set of parameters. The dependencies of proton current on geometry, temperature, and other parameters are examined.
\end{abstract}

Keywords: mitochondrial respiratory chain; proton-pumping Complex I; conformation-assisted transport; proton-coupled electron transfer; quantum yield

\section{Introduction}

The respiratory electron-transport chain of the inner mitochondrial membrane enables eukaryotic cells to store chemical energy from nutrients in the form of adenosine triphosphate (ATP), which serves as the "energy currency" of the cell [1]. The conversion of food energy requires the excitation of highly energetic electrons above $1 \mathrm{eV}$. This excess energy will dissipate if not converted to a more stable form. In the first step of ATP synthesis, proton-pumping complexes stockpile this electron energy by generating and maintaining a proton gradient across the membrane, which manifests itself as a proton-motive force (PMF). In the second step, the PMF facilitates a proton current which drives the rotation of a mechanical nanomotor, the ATP synthase enzyme. In the third step, mechanical energy provides the means for ATP synthesis.

Complex I (NADH-quinone oxidoreductase) is the largest and most elaborate enzyme complex of the respiratory chain [2-5]. It serves as the first electron-acceptor for the incoming reducing equivalents in the respiratory chain. The structure of Complex I was resolved recently in a series of X-ray and cryo-electron microscopy experiments [6-12]. Complex I consists of an L-shaped assembly of a hydrophobic arm embedded in the lipid membrane and a hydrophilic peripheral arm, which protrudes into the mitochondrial matrix (Figure 1). Electron transfer from nicotinamide adenine dinucleotide (NADH) to quinone occurs in the hydrophilic domain via a set of FeS complexes, while the four proton pumps are located within the membrane region [13]. The electron and proton pathways are spatially separated (up to $30 \mathrm{~nm}$ from the tip of the electron entry to the terminal proton-pumping subunit). The physical mechanism of the electron-proton energy 
exchange remains elusive. It is commonly accepted that electron transfer events facilitate conformational changes along the complex (manifesting as an electrostatic wave) that lead to proton transfer against the established PMF (about $200 \mathrm{mV}$ across the $3 \mathrm{~nm}$ thin membrane) [2,12,14-16]. In our previous work [17], we revealed the physical mechanism of energy transfer from such an electrostatic wave to a pumped proton. For simplicity, we replaced the electron system in our model with an external periodic force. In the present paper, we complete our model by introducing electron transfer as the cause of conformational changes.

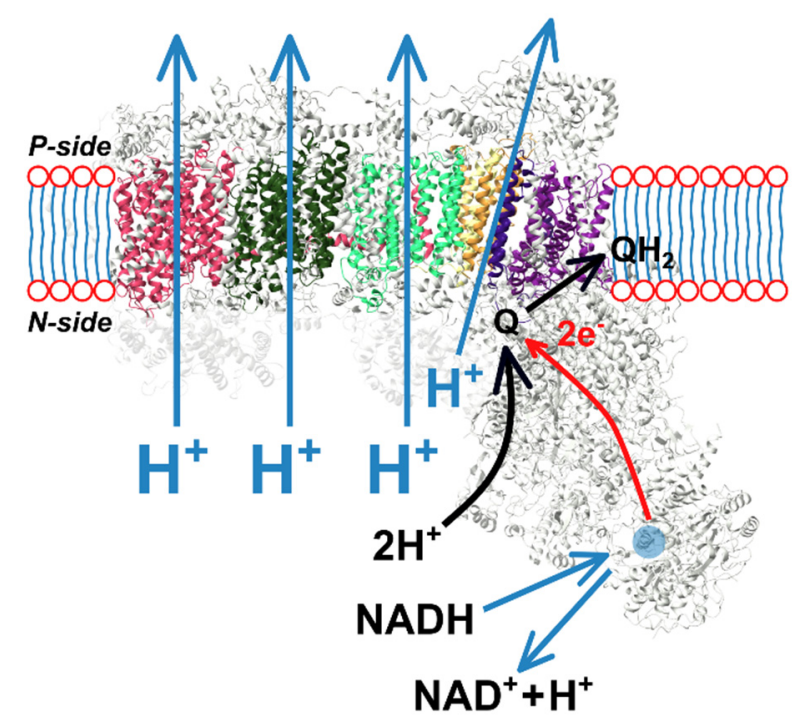

Figure 1. Schematics of proton-pumping Complex I. The protein structure is taken from [10].

Our analysis is based on a single-particle approach exploring resemblances between processes in semiconductor structures and living organisms at the nanoscale. Indeed, within the mitochondrial complexes, electron transport occurs as hopping between metal atoms (or FeS clusters) embedded into the proteins, similar to the hopping between semiconductor quantum dots. Such 0D nanostructures are frequently called "artificial atoms", and we extend the similarity by calling atoms "natural quantum dots". Non-radiative energy transfer process (Förster transfer) in quantum dots is caused by a two-particle electron-hole Coulomb interaction. In proton-pumping complexes, electrostatics is the only possible way to transfer energy from electrons to protons, and this process can also be treated within a two-particle picture. Finally, the temperature for nanostructure operations $(4 \mathrm{~K})$ scales to physiological temperatures of $300 \mathrm{~K}$, similarly to the scale of typical quantum dot energies (few $\mathrm{meV}$ ) to the redox drop from $\mathrm{NADH}$ to $\mathrm{O}_{2}(1.1 \mathrm{eV}$, in several steps). In other words, in biological systems, energy levels are so well separated that they remain discrete even at elevated temperatures. We successfully applied this approach in several papers [17-22] explaining the operating principles of mitochondrial complexes. For many purposes, in physiological conditions, our quantum Heisenberg equations can be rewritten in the form of rate equations with possible quantum effects ignored. When needed, manifestations of quantum effects can be revealed, as in the case of exciton transfer in photosynthetic complexes [23].

\section{Methods}

We examined the model exhibited in Figure 2. It consists of three electron sites placed between two electron reservoirs (source and drain, representing NADH and quinone shuttles, respectively), and three proton sites placed between two proton reservoirs representing positive and negative sides of the membrane. The respective positions of the energy levels demonstrate that electron transport occurs in a usual way, from higher potential to lower, whereas protons are pumped to higher potential. As the energies of electron 
site $L$ and proton site $A$ are below their corresponding chemical potentials, these sites are initially populated from source reservoir and the negative side of the membrane, respectively (Figure 2a). The electron can proceed to the next site $C$ where it is temporarily stuck because of the large energy mismatch between this site and the next site $R$. At the central site, the electron facilitates conformational changes, which we model as a piston with positive charges at the edges. The positive charge at the right edge is attracted by the electron at the central site, and the piston moves to the right (Figure 2b). When the piston is shifted to the right, the energy of the middle proton site $M$ decreases, facilitating proton transfer to this site. Simultaneously, the energy of electron site $C$ drops, decreasing the energy mismatch, and the electron can proceed to site $R$ (Figure $2 \mathrm{c}$ ) and to the drain reservoir. When the electron escapes, elastic forces return the piston to the left, increasing the energy of populated proton site $M$, allowing the proton to move to site $B$ (Figure 2d) and to the positive side of the membrane. Consequently, the mechanical motion of the piston mediates energy transfer from the electronic system to the protonic one. It should be noted that our model is quite simplified in comparison to the real structure. Electron transport occurs via eight $\mathrm{FeS}$ complexes and protons are transferred via various residues and water molecules. We included just three sites in both cases, using two of them for the prevention of back current and allowing for the energy modulation of the middle ones. However, in our simple model, we succeeded in revealing the physical principles both for the creation of the electrostatic wave and for its action into facilitating proton pumping.

To describe this model, we introduce the Hamiltonian:

$$
H=H_{E}+H_{P}+H_{e n v} .
$$

The electron part of the Hamiltonian, $H_{E}$, is given by

$$
\begin{aligned}
H_{E}= & E_{L} a_{L}^{+} a_{L}+E_{C} a_{C}^{+} a_{C}+E_{R} a_{R}^{+} a_{R}+\sum_{k} E_{S k} a_{S k}^{+} a_{S k}+\sum_{k} E_{D k} a_{D k}^{+} a_{D k}-\Delta_{L C} a_{C}^{+} a_{L}-\Delta_{L C}^{*} a_{L}^{+} a_{C} \\
& -\Delta_{R C} a_{C}^{+} a_{R}-\Delta_{R C}^{*} a_{R}^{+} a_{C}-\sum_{k} T_{S k} a_{L}^{+} a_{S k}-\sum_{k} T_{D k} a_{R}^{+} a_{D k}-\sum_{k} T_{S k}^{*} a_{S k}^{+} a_{L}-\sum_{k} T_{D k}^{*} a_{D k}^{+} a_{R}
\end{aligned}
$$

where $a_{\sigma}^{+} / a_{\sigma}$ are the electron creation/annihilation operators for the $\sigma$-site $(\sigma=L, C, R)$ with $E_{\sigma}$ being the electron energies at these sites; $a_{S k}^{+} / a_{S k}$ and $a_{D k}^{+} / a_{D k}$ are the electron creation/annihilation operators for the source and drain, respectively, with $E_{S k}$ and $E_{D k}$ being the energies for these electrons; $\Delta_{\sigma \sigma^{\prime}}$ are the transfer amplitudes between the sites, where $T_{S k}$ and $T_{D k}$ are the amplitudes of the transfers from the source and drain, respectively, to the corresponding electron sites. The proton Hamiltonian has a similar form given by

$$
\begin{gathered}
H_{P}=E_{A} b_{A}^{+} b_{A}+E_{M} b_{M}^{+} b_{M}+E_{B} a_{B}^{+} a_{B}+\sum_{q} E_{N q} b_{N q}^{+} b_{N q}+\sum_{q} E_{P q} b_{P q}^{+} b_{P q}-\Delta_{A M} b_{M}^{+} b_{A}-\Delta_{A M}^{*} b_{A}^{+} b_{M} \\
-\Delta_{B M} b_{M}^{+} b_{B}-\Delta_{B M}^{*} b_{B}^{+} a_{M}-\sum_{q} T_{N q} b_{A}^{+} b_{N q}-\sum_{q} T_{P q} b_{B}^{+} b_{P q}-\sum_{q} T_{N q}^{*} b_{N q}^{+} b_{A}-\sum_{q} T_{P q}^{*} b_{P q}^{+} b_{B},
\end{gathered}
$$

where $b_{\tau}^{+} / b_{\tau}$ are the proton creation/annihilation operators for the $\tau$-site $(\tau=A, M, B)$, with $E_{\tau}$ being the proton energies at these sites; $b_{N q}^{+} / b_{N q}$ and $b_{P q}^{+} / b_{P q}$ are the proton creation/annihilation operators for the negative and positive sides of the membrane, respectively, with $E_{N q}$ and $E_{P q}$ being the energies for these protons; and $\Delta_{\tau \tau^{\prime}}$ are the transfer amplitudes between the sites, where $T_{N q}$ and $T_{P q}$ are the amplitudes of the transfers from the negative and positive sides of the membrane, respectively, to the corresponding proton sites. $H_{e n v}$ describes the coupling of electrons and protons to the protein environment, represented by the set of independent harmonic oscillators, as

$$
H_{P}=\sum_{j} \frac{p_{j}^{2}}{2 m_{j}}+\sum_{j} \frac{m_{j} \omega_{j}^{2}}{2}\left(x_{j}-c_{L j} a_{L}^{+} a_{L}-c_{C j} a_{C}^{+} a_{C}-c_{R j} a_{R}^{+} a_{R}-C_{A j} b_{A}^{+} b_{A}-C_{M j} b_{M}^{+} b_{M}-C_{B j} b_{B}^{+} b_{B}\right)^{2} .
$$


Here, $p_{j}$ and $x_{j}$ are the momentum and coordinate of the $j$-th harmonic oscillator with mass $m_{j}$ and frequency $\omega_{j}$, and $c_{\sigma j}$ and $C_{\tau j}$ are the electron- and proton-environment coupling strengths, respectively.

The energies of the middle electron $(C)$ and proton $(M)$ sites depend on the position of the piston, $x$, as

$$
E_{C}=E_{C 0}-\frac{e^{2}}{4 \pi \varepsilon \varepsilon_{0}} \frac{1}{\sqrt{\left(l_{e}-x\right)^{2}+r_{e}^{2}}}
$$

and

$$
E_{M}=E_{M 0}+\frac{e^{2}}{4 \pi \varepsilon \varepsilon_{0}} \frac{1}{\sqrt{\left(l_{p}+x\right)^{2}+r_{p}^{2}}},
$$

where $l_{e, p}$ and $r_{e, p}$ are the horizontal separations of the piston from the corresponding sites (along the direction of the motion) at the equilibrium position and the vertical shifts, respectively.

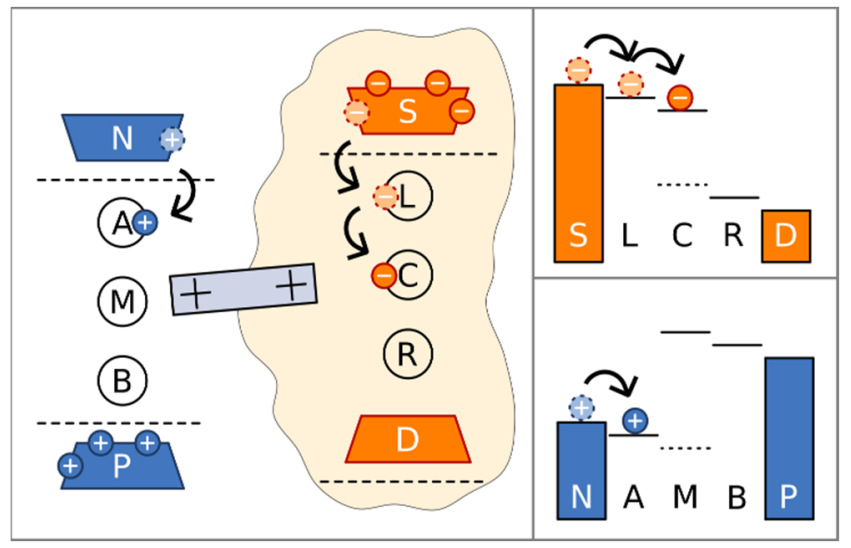

(a)

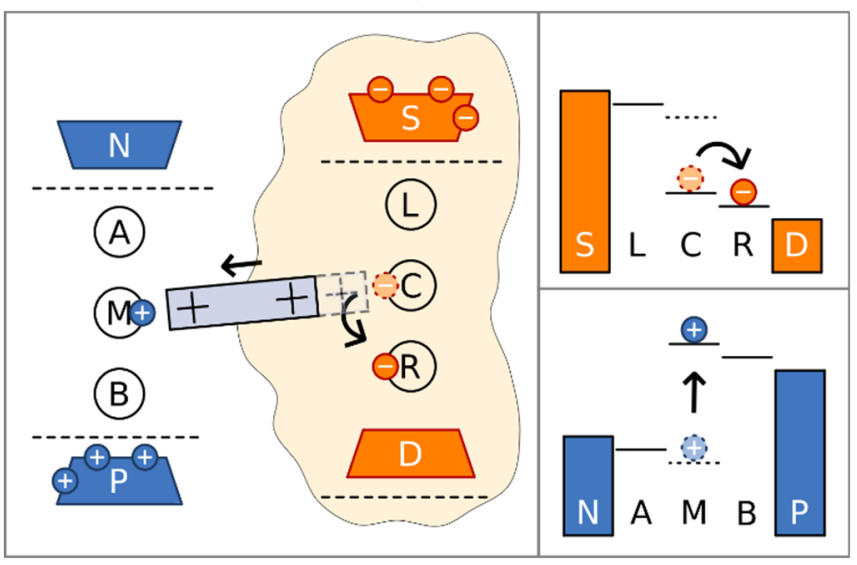

(c)

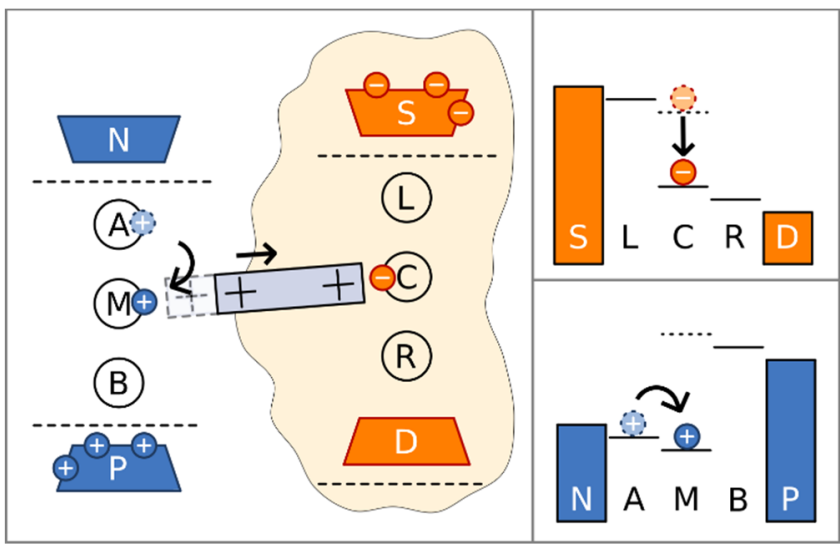

(b)

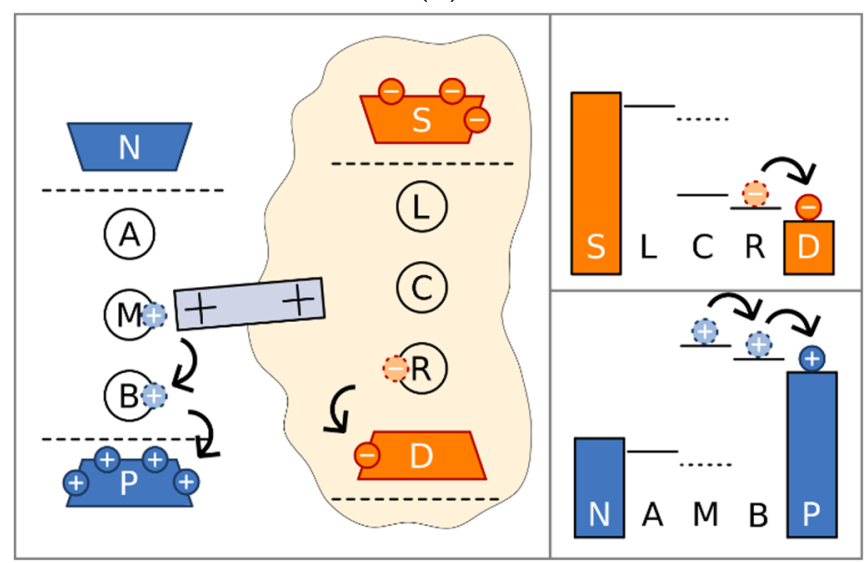

(d)

Figure 2. Piston position, sites populations, and energy levels in the sequence of events leading to the proton pumping. (a) Proton site $A$ and electron site $L$ are populated. Electron proceeds to site $C$. Piston is in the left position. (b) Piston moves to the right attracted by the electron at site $C$. Energies of the electron site $C$ and proton site $M$ decrease. Proton site $M$ is populated. (c) Electron proceeds to site $R$ releasing the piston. Piston moves to the left increasing the energy of the proton site $M$. (d) Proton proceeds to the site $B$ and to the positive side of the membrane. Electron proceeds to the drain reservoir. 
We assume that the piston is in the overdamped regime, and its dynamics can be described by the phenomenological Langevin equation given by

$$
\varsigma \frac{d x}{d t}=-k x+\frac{e^{2}}{4 \pi \varepsilon \varepsilon_{0}} \frac{n_{C}\left(l_{e}-x\right)}{\left(\left(l_{e}-x\right)^{2}+r_{e}^{2}\right)^{3 / 2}}+\frac{e^{2}}{4 \pi \varepsilon \varepsilon_{0}} \frac{N_{M}\left(l_{p}+x\right)}{\left(\left(l_{p}+x\right)^{2}+r_{e}^{2}\right)^{3 / 2}}+\xi(t),
$$

where $\zeta$ is the drag coefficient; the first term on the right side is responsible for the elastic forces returning the piston back to the equilibrium position; the second and third terms are the electrostatic forces from the electrons and protons of the middle sites, respectively, with $n_{C}$ and $N_{M}$ being the populations of these sites; and the last term represents white noise with zero mean and correlation function

$$
\left\langle\xi(t) \xi\left(t^{\prime}\right)\right\rangle=2 \varsigma T \delta\left(t-t^{\prime}\right)
$$

The equations of motion for the electron and proton operators can be obtained from the corresponding Hamiltonians. It was shown previously $[16,19,22]$ that in physiological conditions they can be rewritten in terms of the rate equations for the site populations averaged over the environment. In our model, these equations have forms

$$
\begin{gathered}
\left\langle\dot{n}_{L}\right\rangle+\gamma_{S}\left\langle n_{L}\right\rangle=\gamma_{S} f_{S}\left(E_{L}\right)+\Phi_{L} \\
\left\langle\dot{n}_{R}\right\rangle+\gamma_{D}\left\langle n_{R}\right\rangle=\gamma_{D} f_{D}\left(E_{R}\right)+\Phi_{R} \\
\left\langle\dot{n}_{C}\right\rangle=-\Phi_{L}-\Phi_{R}
\end{gathered}
$$

for electrons, and

$$
\begin{gathered}
\left\langle\dot{N}_{A}\right\rangle+\Gamma_{N}\left\langle N_{A}\right\rangle=\Gamma_{N} F_{N}\left(E_{A}\right)+\Phi_{A}, \\
\left\langle\dot{N}_{B}\right\rangle+\Gamma_{P}\left\langle N_{B}\right\rangle=\Gamma_{P} F_{P}\left(E_{B}\right)+\Phi_{B}, \\
\left\langle\dot{N}_{M}\right\rangle=-\Phi_{A}-\Phi_{B}
\end{gathered}
$$

for protons. Here, the angular brackets mean both thermal and quantum-mechanical averaging. The reservoir coupling constants are calculated as

$$
\begin{aligned}
\gamma_{S / D} & =\sum_{k} T_{S / D k} \delta\left(\omega-E_{S / D k}\right), \\
\Gamma_{N / P} & =\sum_{q} T_{N / P q} \delta\left(\omega-E_{N / P q}\right)
\end{aligned}
$$

and assumed to be frequency-independent. The reservoir distribution Fermi functions are given by

$$
\begin{aligned}
f_{S / D}\left(E_{L / R}\right) & =\left(\exp \left\{\left(E_{L / R}-\mu_{S / D}\right) / T\right\}+1\right)^{-1}, \\
F_{N / P}\left(E_{A / B}\right) & =\left(\exp \left\{\left(E_{A / B}-\mu_{N / P}\right) / T\right\}+1\right)^{-1},
\end{aligned}
$$

where $\mu_{S, D . N, P}$ are the chemical potentials of the corresponding reservoirs. Kinetic coefficients $\Phi_{\sigma}(\sigma=L, R)$ and $\Phi_{\tau}(\tau=A, B)$ have the forms

$$
\begin{gathered}
\Phi_{\sigma}=\kappa_{\sigma}\left(E_{\sigma}-E_{C}+\lambda_{\sigma}\right)\left\langle n_{C}\right\rangle\left\langle 1-n_{\sigma}\right\rangle-\kappa_{\sigma}\left(E_{C \sigma}-E+\lambda_{\sigma}\right)\left\langle n_{\sigma}\right\rangle\left\langle 1-n_{C}\right\rangle, \\
\Phi_{\tau}=\kappa_{\tau}\left(E_{\tau}-E_{M}+\Lambda_{\tau}\right)\left\langle N_{M}\right\rangle\left\langle 1-N_{\tau}\right\rangle-\kappa_{\tau}\left(E_{M}-E_{\tau}+\Lambda_{\tau}\right)\left\langle N_{\tau}\right\rangle\left\langle 1-N_{M}\right\rangle,
\end{gathered}
$$

where

$$
\begin{aligned}
& \kappa_{\sigma}(E)=\left|\Delta_{\sigma C}\right|^{2} \sqrt{\frac{\pi}{\lambda_{\sigma} T}} \exp \left\{-\frac{E^{2}}{4 \lambda_{\sigma} T}\right\}, \\
& \kappa_{\tau}(E)=\left|\Delta_{\tau M}\right|^{2} \sqrt{\frac{\pi}{\Lambda_{\tau} T}} \exp \left\{-\frac{E^{2}}{4 \Lambda_{\tau} T}\right\}
\end{aligned}
$$

are the Marcus rates, and

$$
\begin{aligned}
& \lambda_{\sigma}=\sum_{j} \frac{m_{j} \omega_{j}}{2}\left(c_{\sigma j}-c_{C j}\right)^{2} \\
& \Lambda_{\tau}=\sum_{j} \frac{m_{j} \omega_{j}}{2}\left(C_{\tau j}-C_{M j}\right)^{2}
\end{aligned}
$$


are the reorganization energies of the environment due to electron and proton transfer events. The Marcus rates are not postulated here but derived microscopically $[16,19,22]$.

When electron and proton populations are determined, the corresponding currents can be calculated. As expected, the incoming and outgoing currents are equal in magnitude and opposite in sign for protons and electrons and given by

$$
\begin{gathered}
I_{\mathcal{e}}=\frac{d}{d t} \sum_{k} a_{S k}^{+} a_{S k}=\gamma_{S}\left(n_{L}-f_{S}\left(E_{L}\right)\right), \\
I_{p}=\frac{d}{d t} \sum_{q} b_{N q}^{+} b_{N q}=\Gamma_{N}\left(N_{A}-F_{N}\left(E_{A}\right)\right) .
\end{gathered}
$$

Correspondingly, the quantum yield of the system is defined as the ratio of the proton and electron current magnitudes,

$$
Q Y=\left|I_{p}\right| /\left|I_{e}\right| .
$$

\section{Results}

We solved the set of coupled equations, Equations (7), (9), and (10), numerically using the MATLAB software package [24]. To determine the system parameters for numerical procedure, we held constant known values from the literature. In particular, in order for the voltage across the membrane to be $160 \mathrm{mV}$, we fixed the proton chemical potentials at $\mu_{N}=-80 \mathrm{meV}$ and $\mu_{, P}=80 \mathrm{meV}$, while the energies of the proton sites were $E_{A}=-60 \mathrm{meV}$, $E_{B}=100 \mathrm{meV}$, and $E_{M 0}=-150 \mathrm{meV}$. The drag coefficient, $\zeta=4.14 \mathrm{nN} \mathrm{m}^{-1} \mathrm{~s}$, corresponds to the diffusion coefficient $D=T / \zeta=10^{-12} \mathrm{~m}^{2} \mathrm{~s}^{-1}$, known for lipid membranes [25]. The reorganization energy associated with the electron transfer between the FeS complexes is $\lambda_{\sigma}=700 \mathrm{meV}$ [26]. As the proton coupling to the environment is weaker, $\Lambda_{\tau}=50 \mathrm{meV}$. For the other parameters, we generated a cost-function and used fmincon to find the optimal parameters for maximal proton-pumping current. In this, the inter-site transfer amplitudes and the coupling to reservoirs were initially assumed to be about $1 \mathrm{meV}$ and $0.01 \mathrm{meV}$, respectively. The resulting optimal values were $\Delta_{A M}=0.42 \mathrm{meV}, \Delta_{B M}=0.31 \mathrm{meV}$, $\Delta_{L C}=0.7 \mathrm{meV}, \Delta_{R C}=0.45 \mathrm{meV}, \Gamma_{N}=0.0052 \mathrm{meV}, \Gamma_{P}=0.0047 \mathrm{meV}, \gamma_{S}=0.059 \mathrm{meV}$, and $\gamma_{D}=0.026 \mathrm{meV}$

The obtained time dependencies of piston position as well as electron and proton site populations are shown in Figures 3 and 4, respectively. One can see that the piston motion exhibits noisy dynamics superimposed on quasiperiodic motion caused by electrostatic forces from populated middle sites. The same periodicity can be seen in the proton population as well. Larger electron reorganization energy leads to smoother dynamics. Both electrostatic forces on the piston from electrons and protons are exerted to the right (see Figure 2). Correspondingly, a new equilibrium position is set near $4 \mathrm{~nm}$ and the piston oscillates around this point.

To obtain the proton-pumping current, we performed both time averaging and averaging over various realizations of white noise. The temperature dependence of this current and its dependence on the energy of the middle proton site are shown in Figure 5. 


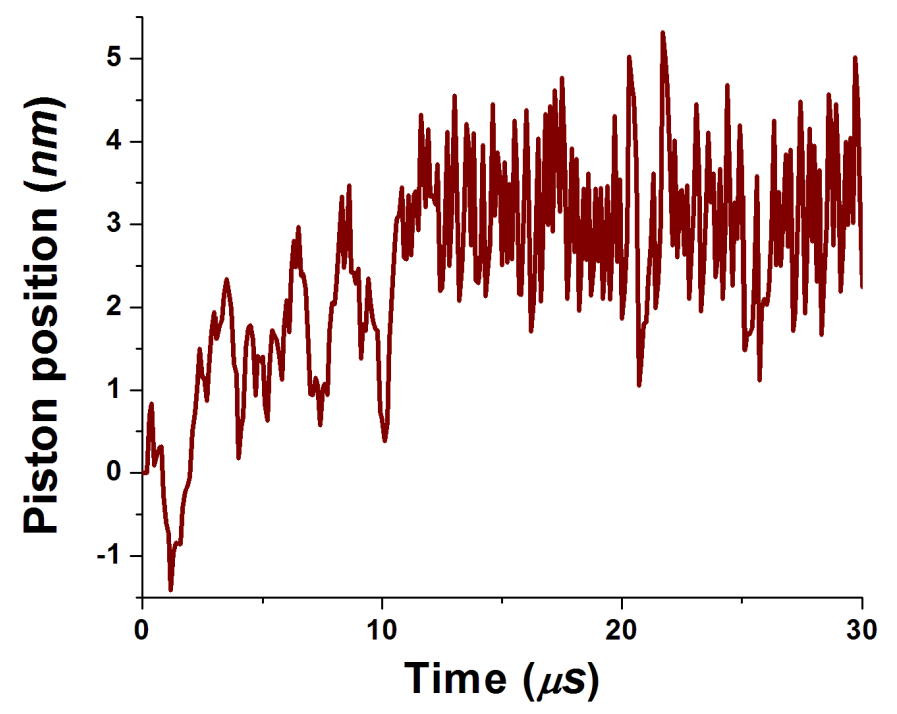

Figure 3. Time dependence of the piston position.

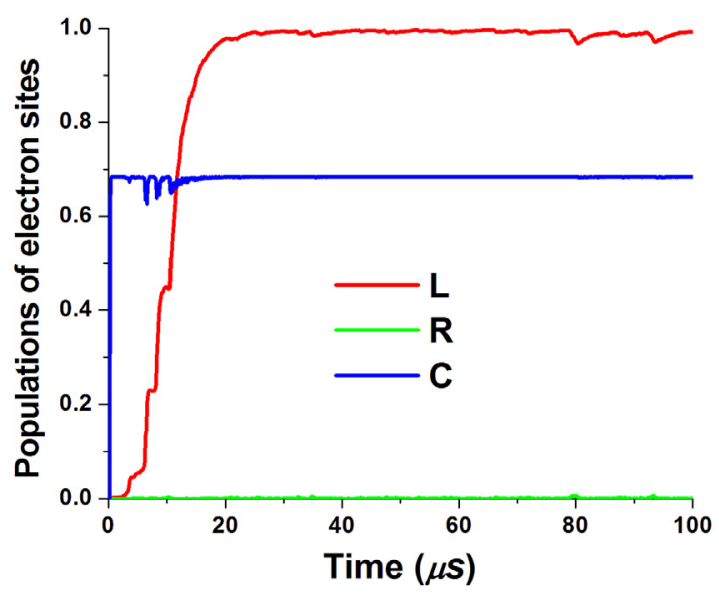

(a)

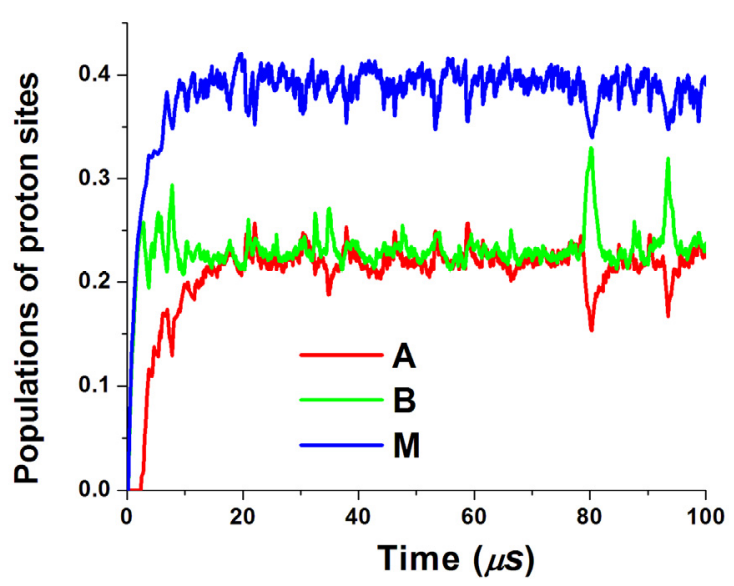

(b)

Figure 4. Time dependencies of site populations. (a) Populations of electron sites $L, R$, and $C$; (b) populations of proton sites $A, B$, and $M$.

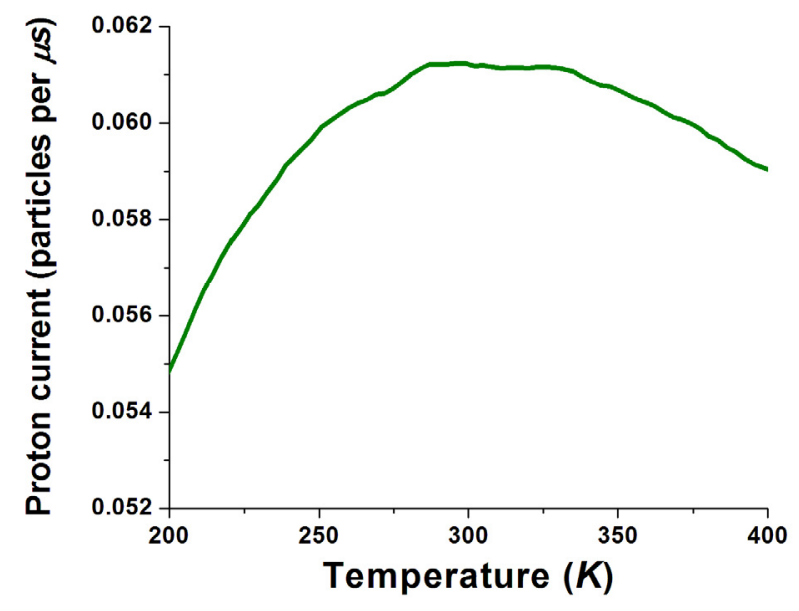

(a)

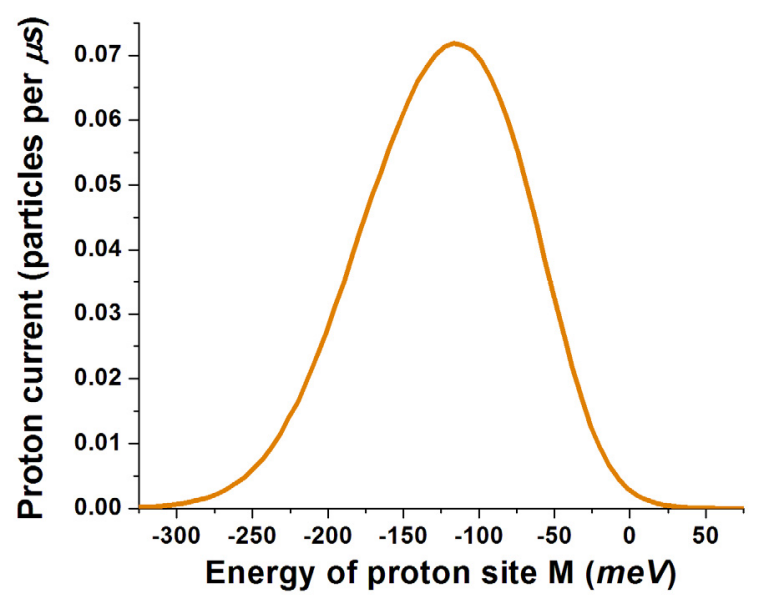

(b)

Figure 5. Proton-pumping current: (a) as a function of temperature; (b) as a function of the energy of the proton middle site. 
It is evident from Figure $5 \mathrm{a}$ that for the present set of parameters, proton pumping is most effective at physiological temperatures. Site $M$ dependence (Figure $5 b$ ) exhibits clear resonant character with maximal value at $-100 \mathrm{meV}$ and drops to zero when the energy level is outside the working regime for the model of Figure 2.

We examined the geometrical conditions for the functionality of our model by plotting the dependencies of the currents on separations of the piston equilibrium position from the electron and proton sites in Figure 6. When these separations are large enough, the equations for particle operators and for piston position become decoupled; the electron and proton parts of the system are separated, and there is no more energy transfer between them. Accordingly, the proton-pumping current should vanish. Indeed, when the separation between the piston and proton site $M$ increases, this current decreases monotonically (see Figure 6a). However, the dependence of the proton current on the separation between the piston and electron site $C$ reaches a maximum when control of the piston dynamics by the site population is the most efficient.

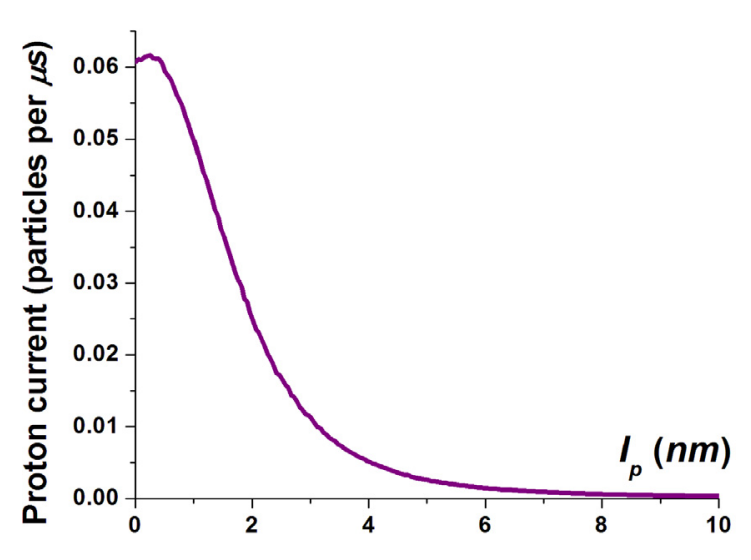

(a)

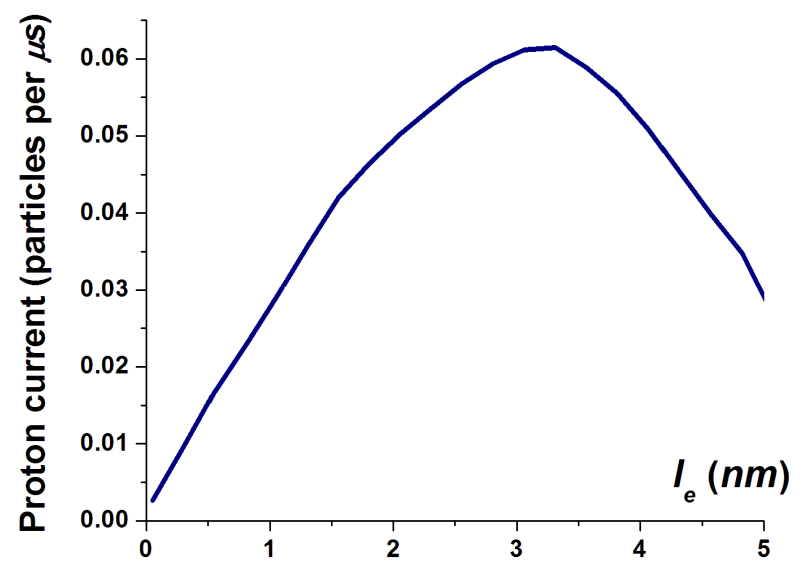

(b)

Figure 6. Dependencies of proton-pumping current on the separation of equilibrium piston position with respect to (a) proton site $M$ and (b) electron site $C$.

\section{Discussion}

Our results shown in the previous section suggest that the simple physical model of Figure 2 can describe the operating principles and working regimes of the protonpumping Complex I of the mitochondrial respiratory chain. We found that the pumping of protons against the concentration gradient can be achieved by means of conformationmediated energy supply from electrons. For a reasonable set of parameters, maximal proton-pumping current occurs at physiological temperatures. However, we believe that our model establishes the underlying physical mechanism of electrostatic wave propagation along the membrane arm of Complex I and the subsequent proton pumping promoted by this wave. If we compare the pumping rate in our model (tens of particles per millisecond) with ATP production in mitochondria (about 100 per second [1]), we can see that our optimal values are several orders of magnitude larger. Correspondingly, even if in the real system, the presently unknown parameters are found to be far away from the optimal values, our model is likely to still be valid.

Our model of Complex I can be combined with our previous models of Complex III and quinone dynamics [22], Complex IV [20], and the rotating $\mathrm{F}_{0}$ motor of the ATP synthase (Complex V) [19]. Investigation of all these subsystems is based on single-particle Heisenberg equations of motion, two-particle Coulomb interaction for the exchange energy, and phenomenological Langevin equations for the moving parts (shuttles and conformation 
changes). We believe that our unified, self-consistent model of mitochondrial respiratory chain functionality provides a coherent framework for experimental and theoretical researchers studying energy transduction in eukaryotic cells in detail.

Author Contributions: Conceptualization, L.M. and M.V.; methodology, L.M.; software, J.F. and L.M.; validation, J.F., L.M. and M.V.; formal analysis, L.M.; investigation, J.F.; resources, J.F. and L.M.; data curation, J.F.; writing—original draft preparation, J.F. and L.M.; writing—review and editing, M.V.; visualization, J.F. and L.M.; supervision, L.M.; project administration, M.V.; funding acquisition, L.M. and M.V. All authors have read and agreed to the published version of the manuscript.

Funding: This research was funded by AFOSR, Award No FA9550-16-1-0279.

Institutional Review Board Statement: Not applicable.

Data Availability Statement: Not applicable.

Acknowledgments: L.M. is grateful to Anatoly Smirnov for numerous helpful discussions.

Conflicts of Interest: The authors declare no conflict of interest.

\section{References}

1. Alberts, B.; Alberts, B.; Johnson, A.; Lewis, J.; Raff, M.; Roberts, K.; Walter, P. Molecular Biology of the Cell; Garland Science: New York, NY, USA, 2002.

2. Verkhovskaya, M.; Bloch, D.A. Energy-converting respiratory Complex I: On the way to the molecular mechanism of the proton pump. Int. J. Biochem. Cell. Biol. 2013, 45, 491. [CrossRef] [PubMed]

3. Hirst, J. Mitochondrial complex I. Annu. Rev. Biochem. 2013, 82, 551-575. [CrossRef] [PubMed]

4. Sazanov, L.A. A giant molecular proton pump: Structure and mechanism of respiratory complex I. Nat. Rev. Mol. Cell Biol. 2015, 16, 375-388. [CrossRef]

5. Ohnishi, T.; Ohnishi, S.T.; Salerno, J.C. Five decades of research on mitochondrial NADH-quinone oxidoreductase (complex I). Biol. Chem. 2018, 399, 1249-1264. [CrossRef]

6. Baradaran, R.; Berrisford, J.M.; Minhas, G.S.; Sazanov, L.A. Crystal structure of the entire respiratory complex I. Nature 2013, 494, 443. [CrossRef] [PubMed]

7. Zickermann, V.; Wirth, C.; Nasiri, H.; Siegmund, K.; Schwalbe, H.; Hunte, C.; Brandt, U. Mechanistic insight from the crystal structure of mitochondrial complex I. Science 2015, 347, 44. [CrossRef]

8. Zhu, J.; Vinothkumar, K.R.; Hirst, R. Structure of mammalian respiratory complex I. Nature 2016, 536, 354. [CrossRef]

9. Fiedorczuk, K.; Letts, J.A.; Degliesposti, G.; Kaszuba, K.; Skehel, M.; Sazanov, L.A. Atomic structure of the entire mammalian mitochondrial complex I. Nature 2016, 538, 406. [CrossRef]

10. Guo, R.; Zong, S.; Wu, M.; Gu, J.; Yang, M. Architecture of Human Mitochondrial Respiratory Megacomplex I2III2IV2. Cell 2017, 170, 1247-1257. [CrossRef]

11. Parey, K.; Haapanen, O.; Sharma, V.; Köfeler, H.; Züllig, T.; Prinz, S.; Siegmund, K.; Wittig, I.; Mills, D.J.; Vonck, J.; et al. High-resolution cryo-EM structures of respiratory complex I: Mechanism, assembly, and disease. Sci. Adv. 2019, 5, eaax9484. [CrossRef]

12. Kampjut, D.; Sazanov, L.A. The coupling mechanism of mammalian respiratory complex I. Science 2020, 370, eabc4209. [CrossRef]

13. Jones, A.J.Y.; Blaza, J.N.; Varghese, F.; Hirst, J. Respiratory complex I in Bos Taurus and Paracoccus denitrificans pumps four protons across the membrane for every NADH oxidized. J. Biol. Chem. 2017, 292, 4987-4995. [CrossRef] [PubMed]

14. Brandt, U. A two-state stabilization-change mechanism for proton-pumping complex I. Biochim. Biophys. Acta 2011, 1807, 1364. [CrossRef] [PubMed]

15. Kaila, V.R.I. Long-range proton-coupled electron transfer in biological energy conversion: Towards mechanistic understanding of respiratory complex I. J. R. Soc. Interface 2018, 15, 20170916. [CrossRef] [PubMed]

16. Zhang, X.C.; Li, B. Towards understanding the mechanisms of proton pumps in Complex-I of the respiratory chain. Biophys. Rep. 2019, 5, 219-234. [CrossRef]

17. Kaur, D.; Filonenko, I.; Mourokh, L.; Fendler, C.; Blick, R.H. Stochastic resonance in a proton pumping Complex I of mitochondria membranes. Sci. Rep. 2017, 7, 12405. [CrossRef]

18. Smirnov, A.Y.; Mourokh, L.G.; Nori, F. Förster mechanism of electron-driven proton pumps. Phys. Rev. E 2008, 77, 011919. [CrossRef] [PubMed]

19. Smirnov, A.Y.; Savel'ev, S.; Mourokh, L.G.; Nori, F. Proton transport and torque generation in rotary biomotors. Phys. Rev. E 2008, 78, 031921. [CrossRef]

20. Smirnov, A.Y.; Mourokh, L.G.; Nori, F. Kinetics of proton pumping in cytochrome c oxidase. J. Chem. Phys. 2009, 130, 235105. [CrossRef] [PubMed]

21. Smirnov, A.Y.; Mourokh, L.G.; Nori, F. Electrostatic models of electron-driven proton transfer across a lipid membrane. J. Phys. Cond. Matt. 2011, 23, 234101. [CrossRef] 
22. Mourokh, L.; Vittadello, M. Physical Model of Proton-Pumping Q-cycle in Respiratory and Photosynthetic Electron Transport Chains. Chem. Phys. 2020, 530, 110638. [CrossRef]

23. Mourokh, L.G.; Nori, F. Energy transfer efficiency in the chromophore network strongly coupled to a vibrational mode. Phys. Rev. E 2015, 92, 052720. [CrossRef] [PubMed]

24. MATLAB R2020b and Optimization Toolbox, Version 9.0; The MathWorks, Inc.: Natick, MA, USA, 2020.

25. Berg, J.M.; Tymoczko, J.L.; Stryer, L. Biochemistry, 5th ed.; W.H. Freeman: New York, NY, USA, 2002.

26. Page, C.C.; Moser, C.C.; Chen, X.; Dutton, P.L. Natural engineering principles of electron tunneling in biological oxidationreduction. Nature 1999, 402, 47-52. [CrossRef] 